\title{
Image Classification and Detection of Insulators using Bag of Visual Words and Speeded up Robust Features
}

\author{
Ayushi Jadia, M.P.S Chawla
}

\begin{abstract}
Electrical substation online monitoring in computer vision technology is based on image processing algorithm to perform visual analysis. This paper presents classification of ceramicand glass insulators through Bag of Visual Words and detection of these insulators by Point Feature Matching. The training image datasets are used for categorization by forming a visual vocabulary while a new unlabeled image from testing image dataset is classify using nearest neighbor classification method for features descriptor. For detection we use Speeded up Robust Features for detecting position of insulator present in cluttered scene image. Matching process is done between test and reference image and decision is made based on similar features. We conducted experiment on insulators to verify the superiority of our proposed method. The proposed method can be used in security, surveillance and inspection system.
\end{abstract}

Keywords- Classification, Bag of Visual Words (BOVW), KNearest Neighbor, Detection, Point Feature matching, Speeded up Robust Features (SURF), Insulators

\section{INTRODUCTION}

Over the years, Image processing and Machine learning techniques gained popularity for the visual analysis of images [1]. Image classification and detection of an object is critical task for online monitoring of power equipment. The insulator is main component for electrical insulation and mechanical support for the transmission line [2, 3]. Local point descriptors and key points are used for imagery data classification and image retrieval $[4,5,6,7,8]$. In this paper, for the classification of ceramic and glass insulators the algorithm used is knowns as (BOVW) Bag of Visual Words.[9, 10].

Image can be analyzed based on their color, texture, shape and edges etc. Different categories of Content based image retrieval (CBIR) methods proposed by D. Latha and Dr. Y. Jacob Vetha Raj[11] gives brief idea about image retrieval process using color, texture, shape and any information extracted for query and database images. Local feature detecting algorithmsuch as Speed Up Robust Feature (SURF) [12] and Scale Invariant Feature Transform (SIFT)[13] are well known descriptors. To find images similar to a test image, all images feature descriptors must be compared using some distance measures. BOVW with SURF features is used for classification.

Feature matching process is widely used for matching features by using several algorithms such as template matching, features descriptors etc.But the fastest features matching algorithm proposed by Zetao Jiang et al gives brief idea about SURF features [14].

Revised Version Manuscript Received on September 15, 2020.

* Correspondence Author

Ayushi Jadia*, Electrical Engineering Department SGSITS, Indore jadiaayushi@gmail.com

M.P.S Chawla, Electrical Engineering Department, SGSITS, Indore mpschawla@gmail.com

(C) The Authors. Published by Blue Eyes Intelligence Engineering and Sciences Publication (BEIESP). This is an open access article under the CC BY-NC-ND license (http://creativecommons.org/licenses/by-nc-nd/4.0/)
The process for detection of insulator is divided into three steps using SURF algorithm which are "Detection step", "Description step", and "Matching step". This algorithm can be used in various exercises such as face recognition, tracking of object, location of object etc. In this paper we use SURF algorithm for detection and location of object from a source image. [15]

\section{II. \\ CLASSIFICATION AND DETECTION OF INSULATORS}

\section{A. Bag of visual words (BOVW)}

Image dataset consist of images of different categories, thus in order to extract features from an image or to detect, extract features and descriptors from all image in the datasets, and construct a visual dictionary. There are several applications and feature extracting algorithms for detecting features and extracting descriptors in an image. Some of the feature extractor algorithms are SIFT, KAZE, SURF etc. $[13,16]$ In this paper we use SURF feature representative for each image category. This is accomplished by:

1. Extracting SURF feature from all the images in all image categories.

2. Constructing the visual vocabulary by diminishing the quantity of features through quantization of feature space utilizing k-means clustering.

Fig.2 show the block diagram representation of algorithm involving the steps which are followed to classify the insulators namely.

The first step is to prepare image dataset based on two categories. The two categories of images have ceramic insulators and glass insulators. After preparing the datasets of these categories SURF features are extracted by feature extraction and then descriptors are extracted to construct the visual vocabulary. These features vocabulary will help in classification of the categories of different features. This algorithm is tested by test image for the classification which are unlabeled images of insulators which is not used in training and validation process. In fig. 2 the block diagram representation of the proposed method of the BOVW (Bag of Visual Words) algorithm is shown.

\section{B. k-Means Clustering}

Clustering is unsupervised iterative method that classify group of points into clusters based on similarity. The similarity measure frequently depending on distance methods e.g. Euclidean distance to classify points in groups (or clusters) [17] K-means clustering algorithm is an unsupervised classification procedure which classifies or groups the objects automatically into $\mathrm{K}$ number of group where each group contain points that have minimum distance between them.

Published By: 
K-means is also called C-means or ISODATA clustering method. K-means algorithm initialize clusters center (or centroids) by selecting samples at random from training vectors. K-means is repeated method which used to collect data into groups and these groups change every iteration.

\section{Speeded up Robust Features (SURF)}

The fast detector and descriptor, called SURF (Speeded up robust features), described in [18]. Object detection and its feature extraction is done by SURF (Speeded Up Robust Feature) algorithm in this project. It depends on multi scale space hypothesis and its feature identifier depend on Hessian Matrix; we rely on the determinant of Hessian also for the Scale section as done by Lindeberg [19]. Hessian matrix has good performance and accuracy as compared to other SIFT algorithmin an image which is define as $I, X=$ $(x, y)$ where $I, X$ isgiven point and Hessian matrix is given by (1). $\mathrm{H}(\mathrm{x}, \sigma)$ in $\mathrm{X}$ at scale $\sigma$, it is defined as

$$
H(x, \sigma)=\left(\begin{array}{ll}
\operatorname{Lx} x(x, \sigma) & \operatorname{Lxy}(x, \sigma) \\
\operatorname{Lyx}(x, \sigma) & \operatorname{Lyy}(x, \sigma)
\end{array}\right)
$$

Here $L_{x x}(x, \sigma)$ is convolutionoutcome of the second order derivative of Gaussian filter with the image $I$ in point $X$ and likewise for $L_{x y}(x, \sigma)$ and $L_{y y}(x, \sigma)$.

Hessian Matrix used in SURF for selection of candidate points in different sizes and usesas is in Hessian Laplace Method. The number of detectors and descriptors variety have been already proposed in the literature (e.g. [19], [20], [21], [22]). It calculates Haar-wavelet responses in $\mathrm{x}$ and $\mathrm{y}$ direction for it to be invariant to rotation shown in fig. 1.

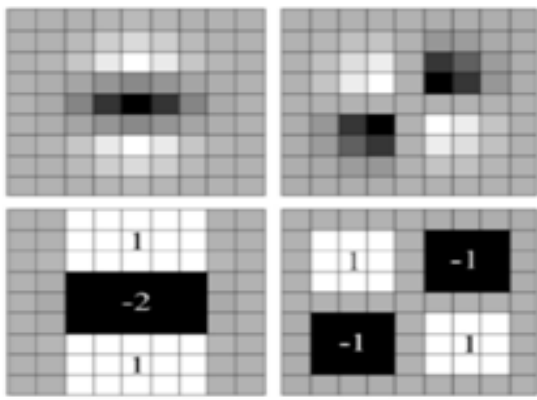

Fig.1 The Gaussian second order partial derivatives in $\mathbf{y}$ direction and $x$-ydirection. [18]

SURF algorithm is able to detect strong point features in an image which is helpful for matching object in order to detect the presence of an object in a given image.

\section{Object Detection}

In the field of image processing object detection plays an important role. The algorithm we used can describe local features of any interest object and features are extracted or descriptor points from it and compare with the reference image that is the original image. Matching process is taking place to find similar feature in image to retrieve that object of interest. The algorithm used is called SURF (Speeded Up Robust Features). Power equipment image captured resembles as a cluttered scene. This algorithm work best for cluttered scene to find out region of interest that is the object. [15] This algorithm is depending on comparing and inspecting points between the reference image and the cluttered scene image. Detection of object in real time is a challenging task. For such time critical situation point feature matching is an appealing solution as object can be learned online instead of statistical learning techniques that requires a lot training sample. [15].

\section{E. SIFT and SURF Comparison}

In the recent years,identificationof objects using descriptors of image havedeveloped considerably and has been excessively encouraging in machine vision technology. Some local features extraction algorithms are available that play a major role in detection of 2D patterns such as face that also used in 3D objects. The most well-known local descriptors are SIFT and SURF that are powerful and capable forvarious machine vision application. In 1999 Lowe [23] represented Scale Invariant Feature Transform (SIFT), the aim of this algorithm is to extract features that are recapitulate. The effect of illumination changes, rotation and image scaling on features are invariant andfor 3D camera viewpoint it is partially invariant. In SURF and SIFT algorithms there is little dissimilarity between techniquesadopted foridentification of features. Visual pyramids made by SIFT to discover candidate points and channels each layer as per the Gauss law with expanded values of sigma and discoverscontrasts. Whereas, SURF utilizes Hessian Matrix [19] to choose the candidate points in various sizes and uses, as is utilized in the HessianLaplace technique[24].

\section{BLOCK DIAGRAM}

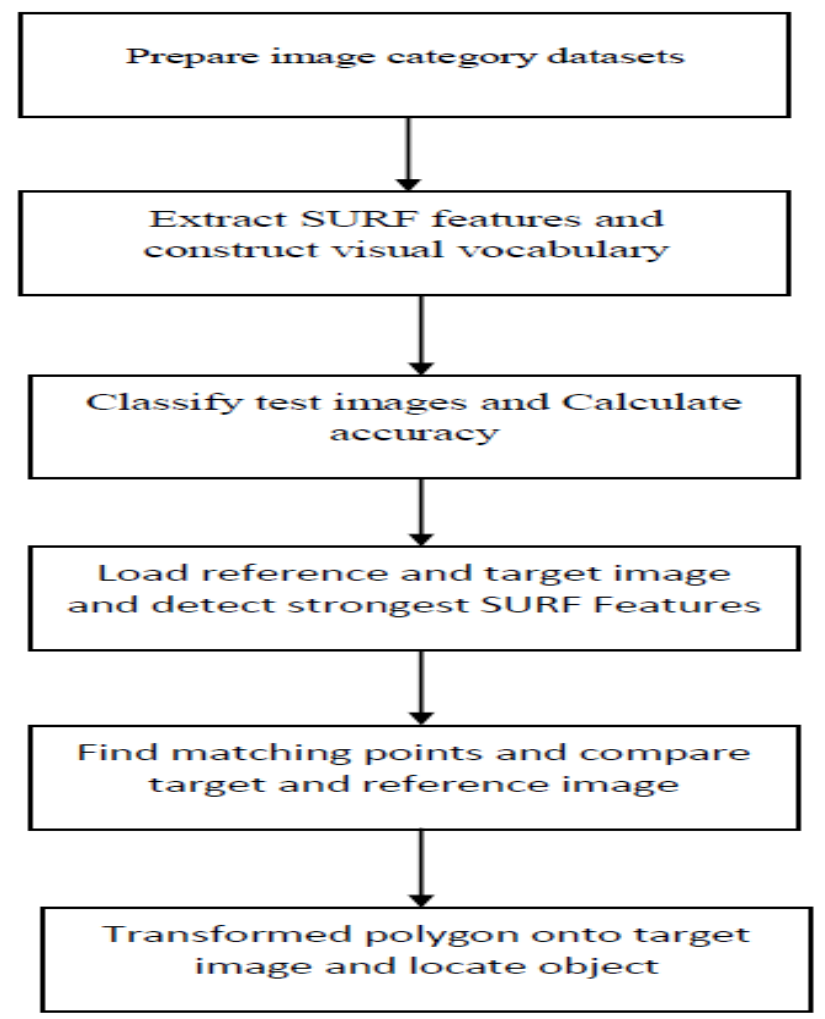

Fig.2 Block diagram of proposed methodology

\section{DATASETS, RESULTS AND DISCUSSION}

\section{A. Datasets}

In this paper ceramic and Glass insulators are used for classification. Fig. 3 show few montage images of Ceramic insulators. Fig. 4 show few images from the image dataset of Glass insulators.

Published By:

Blue Eyes Intelligence Engineering

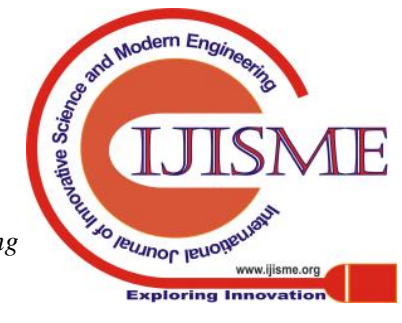


The experimental results of the test image arepresented in a table. Fig. 5 show test images of Ceramic insulator and Glass insulator.

In this work we have taken 500 images in each case total of 1000 images for the image dataset contain ceramic and glass insulators. The test image dataset contains 15 images, 9 glass insulator images and 6ceramic insulators images. The simulation results of the testing images are shown in graph which depict accuracy and sensitivity of correctly classified images. The graph is shown in the fig. 6 .

The Image batch processor tool is used which is available in MATLABR2019a software tool application. By the use of Image Batch Processor, we have converted all the images present in a dataset to $227 * 227$ pixels size. This is done to reduce the size of large image as images quantity that we have taken is 1000 in numbers. Although, the test image dataset consists of different size image for categorization.
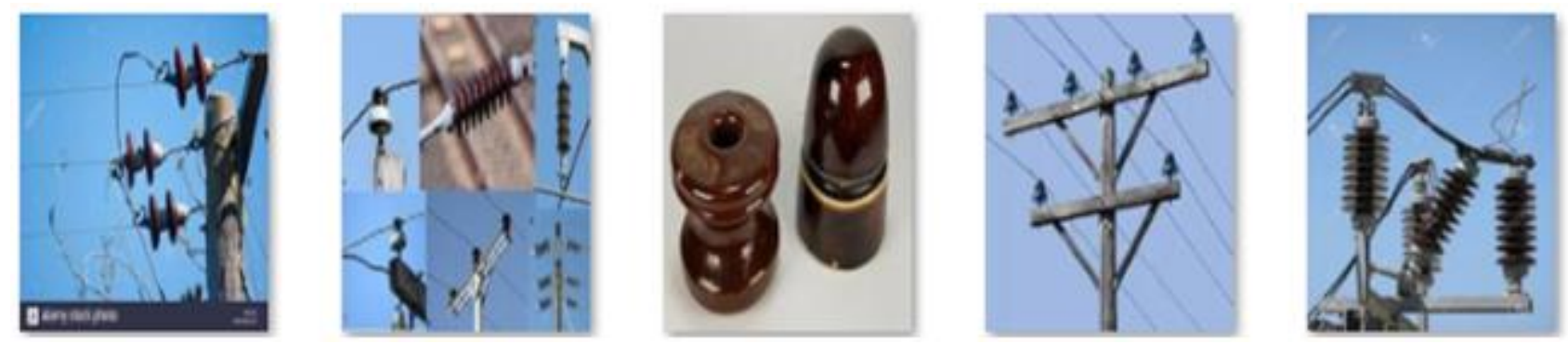

Fig.3 Image dataset of Ceramic insulators
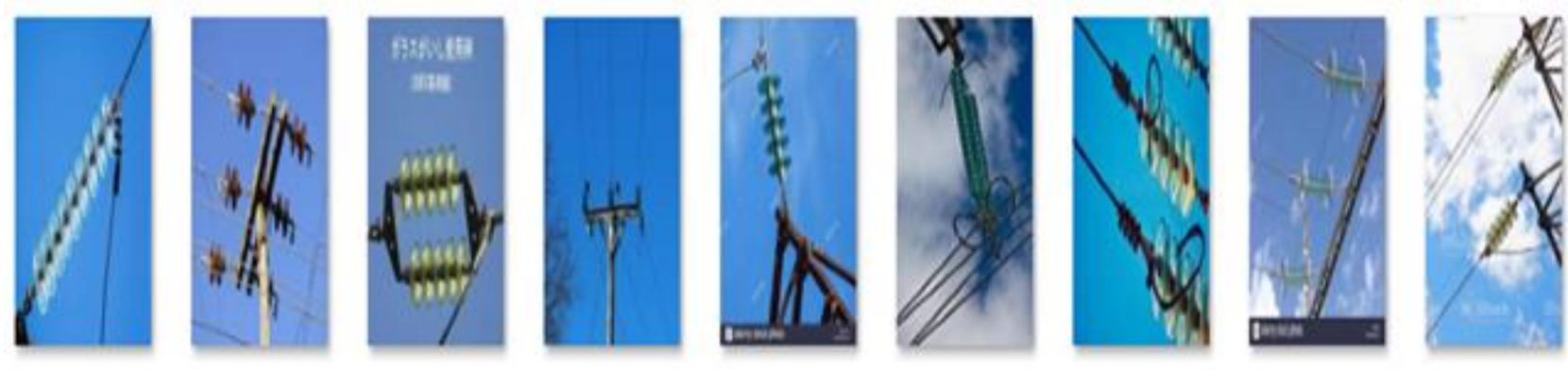

Fig. 4Image dataset of Glass insulators

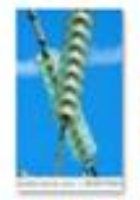

\$1

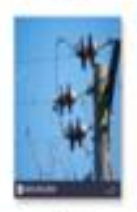

4

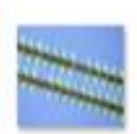

92

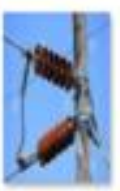

5

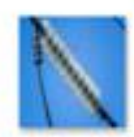

93

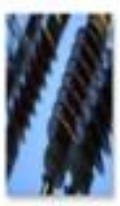

太

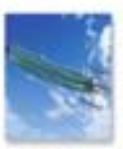

$\phi$

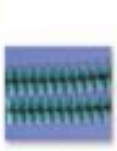

95.

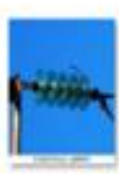

of

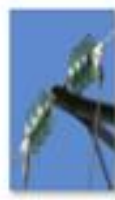

छौ

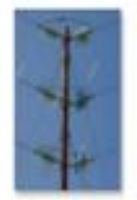

ga

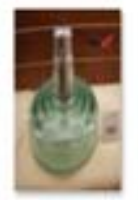

99

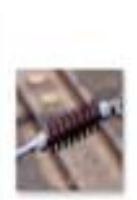

t1

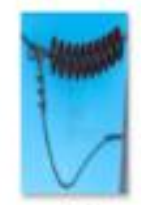

t2

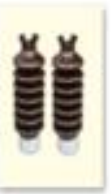

13

Fig. 5 Image dataset of test images

\section{B. Results and Discussion}

The training and validation set average accuracy is evaluated by confusion matrix. From table 1 and 2, we are able to see the number of insulators corresponding to training and validation sets. The average accuracy is also calculated.

Table 1 Confusion Matrix for Training image dataset

\begin{tabular}{|l|l|l|}
\hline \multicolumn{2}{|c}{ PREDICTED } \\
\hline KNOWN & CERAMIC & GLASS \\
\hline CERAMIC & 0.89 & 0.11 \\
\hline GLASS & 0.09 & 0.91 \\
\hline
\end{tabular}


Image Classification and Detection of Insulators using Bag of Visual Words and Speeded up Robust Features

Table 2 Confusion Matrix for Validation image dataset

\begin{tabular}{|l|l|l|}
\hline \multicolumn{2}{|c|}{ PREDICTED } \\
\hline KNOWN & CERAMIC & GLASS \\
\hline CERAMIC & 0.89 & 0.11 \\
\hline GLASS & 0.13 & 0.86 \\
\hline
\end{tabular}

The average accuracy obtained for training set is 0.90 and average accuracy obtained for validation set is 0.88 .

"Quantitative performance of algorithms is reported in terms of sensitivity, specificity. Sensitivity is the fraction of positive class sample correctly classified (the ability of the classifier to find all the positive samples) given by (2). Specificity is the fraction of negative class sample correctly identified. Accuracy is the proportion of true results, either true positive or true negative, in a population given by (3). It measures the degree of veracity of a diagnostic test on a condition." [25]

$$
\text { Sensitivity }=\frac{T p}{T p+F n}
$$

$$
\text { Accuracy }=\frac{T p+T n}{T p+T n+F p+F n}
$$

"TP (true positive): represents the number of images correctly labeled with corresponding class by the algorithm, FP (false positive): represents the number of images not exist in training set but labeled as one of the clusters (unexpected result), FN (false negative): represents the number ofMissing images, TN (true negative): represents the number of images not exist in training set and not labeled correctly." [25]

The figure 6 show the graph between category of insulator and accuracy. The accuracy of glass insulator is obtained as 0.85 and that of ceramic is 0.90 . The sensitivity appears to be same as accuracy for the test images.

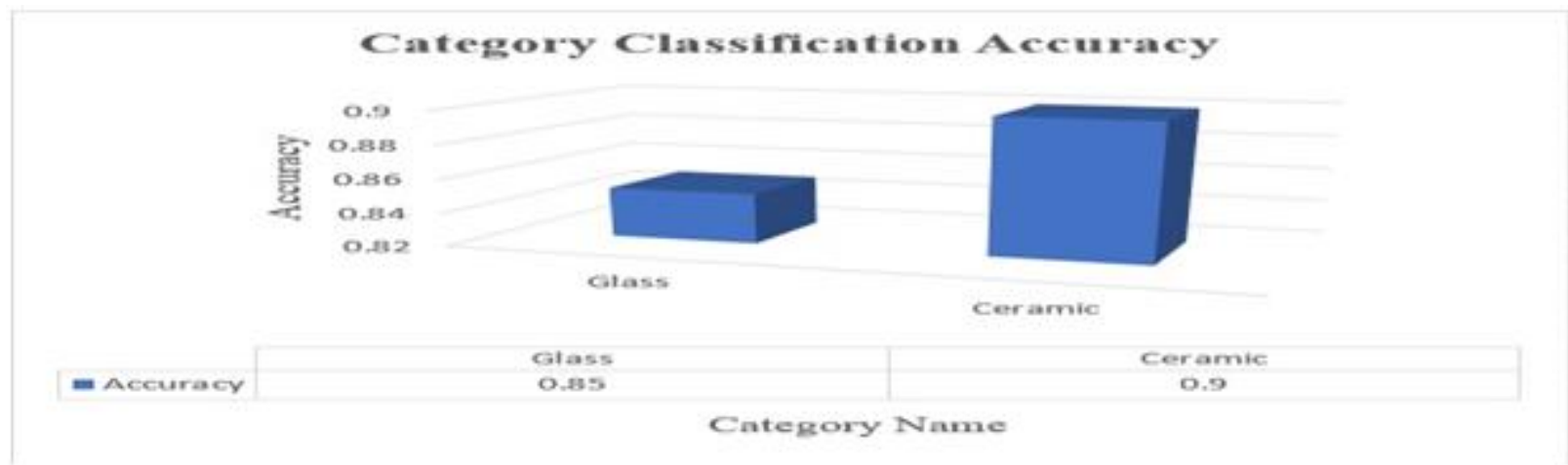

Fig. 6 Graph between Category classification accuracy and category name

Experimental results of point feature matching using SURF are shown in following figures. Fig.7 show the insulator image. This is the reference image which has to be detected in a cluttered scene. Fig. 8 show the cluttered scene image which is a substation image consisting of the reference image. Fig. 9 show 50 strongest feature point detected on a reference image.Fig.10 show 300 strongest feature detection of cluttered scene or target image. Fig. 11 show the match between target and reference incling outliers only. Which show all the matches between the images.
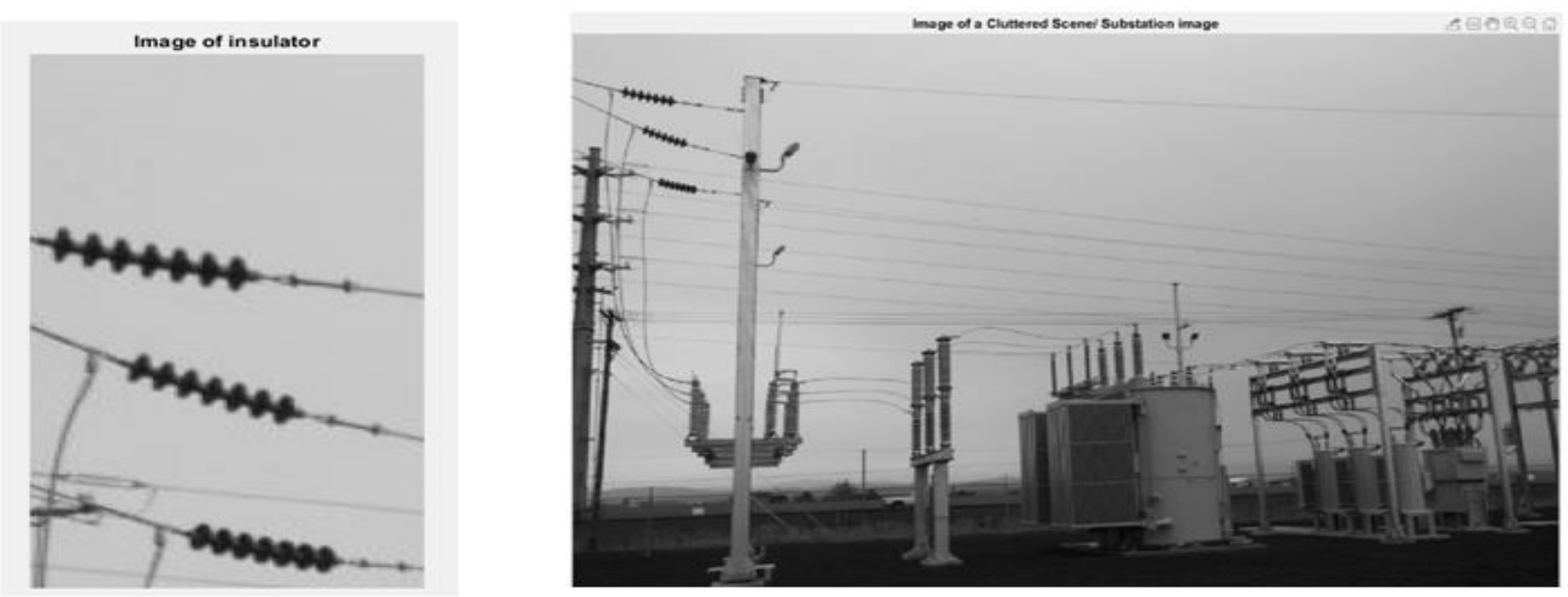

Fig. 7 Image of insulator/ Reference imageFig. 8 Image of a cluttered scene/ Substation image

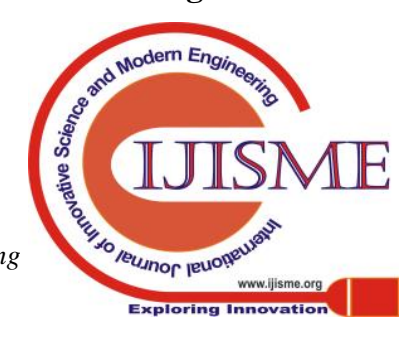



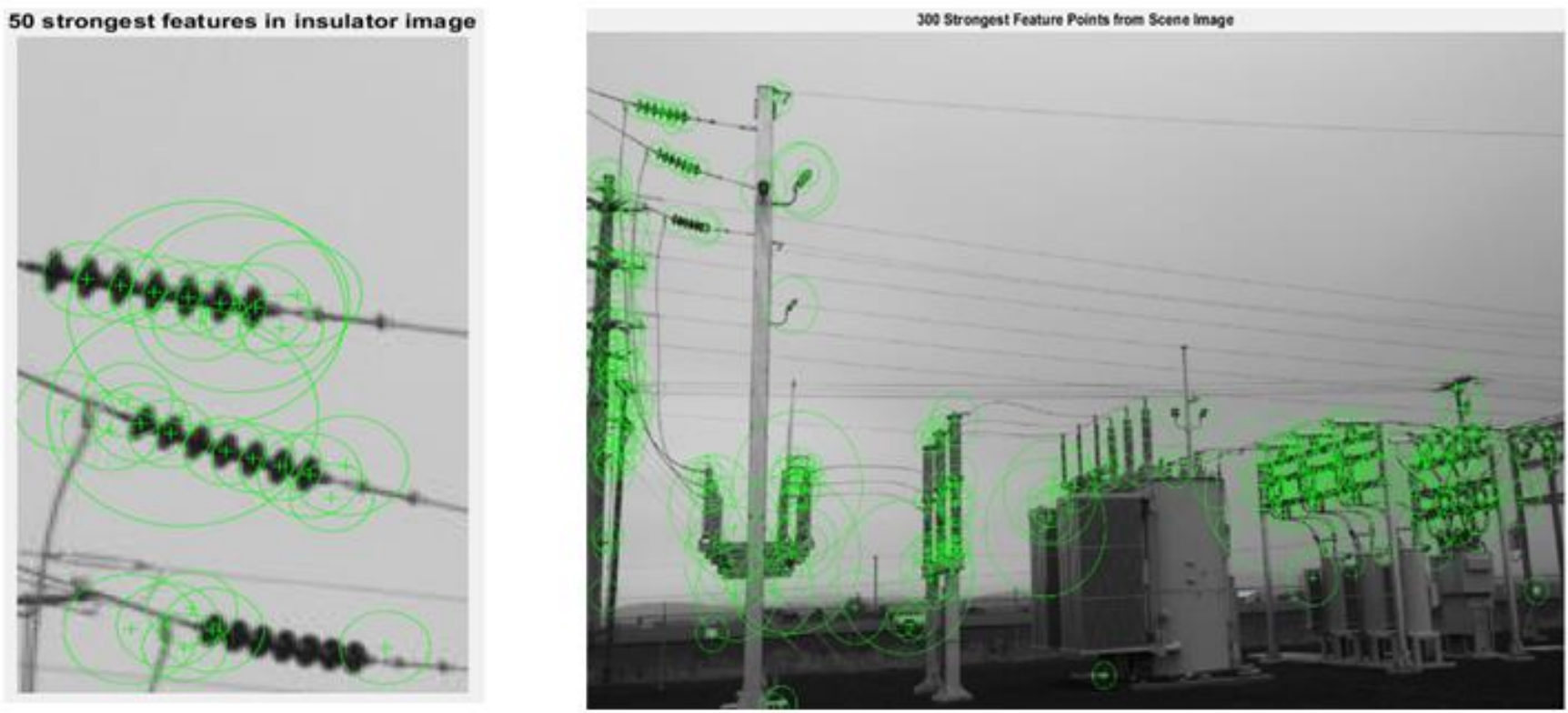

Fig. 950 strongest features in insulator imageFig.10 300 strongest feature points of scene image

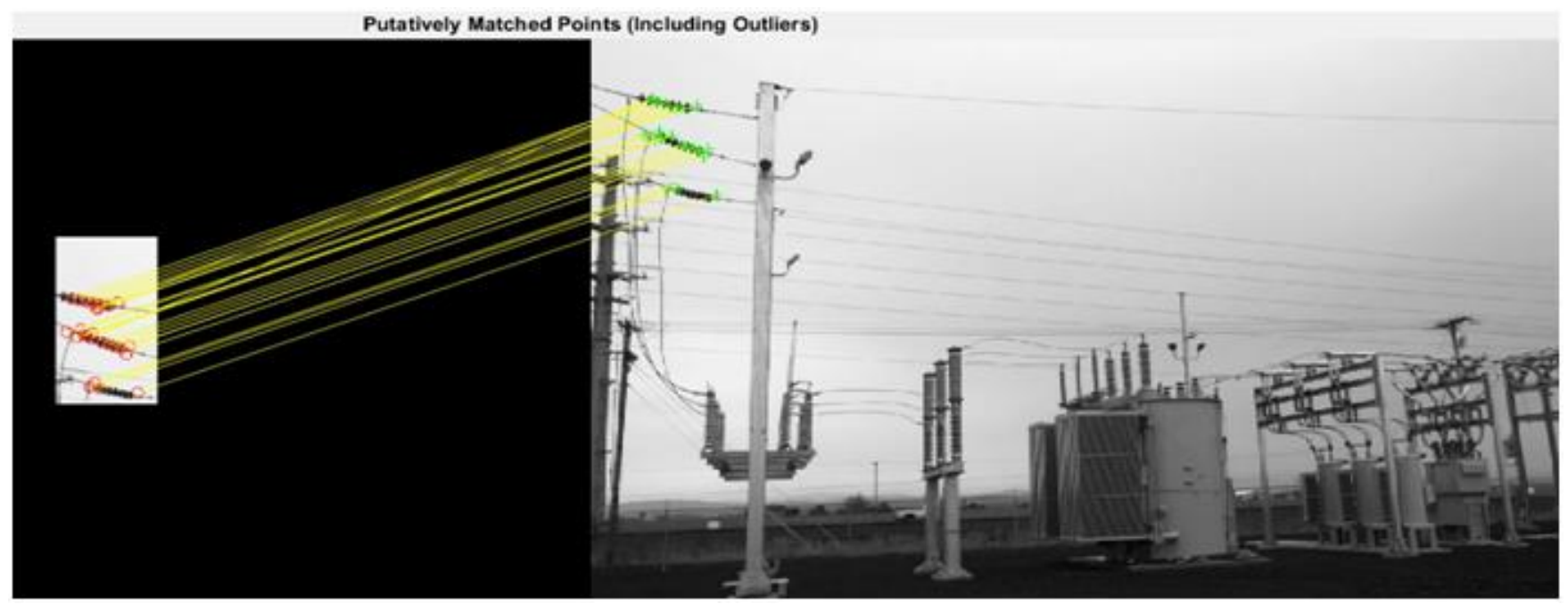

Fig.11 Putative matches including outliers

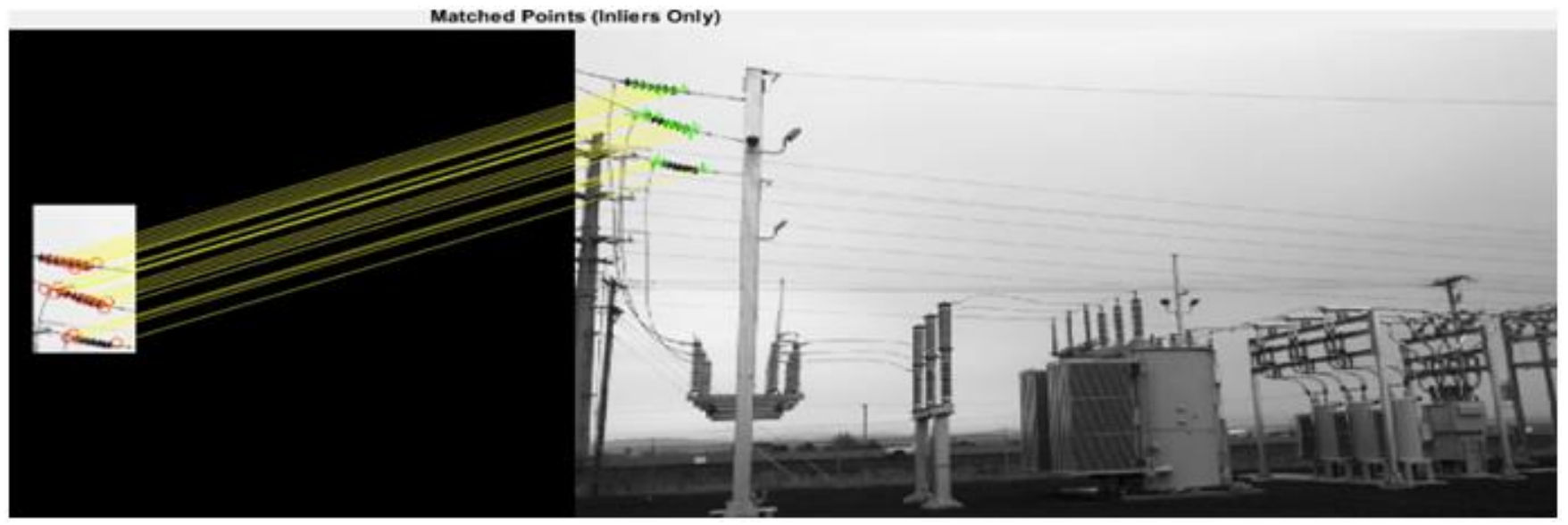

Fig.12 Putative matches including inliers

Published By:

Blue Eyes Intelligence Engineering

\& Sciences Publication

(C) Copyright: All rights reserved.

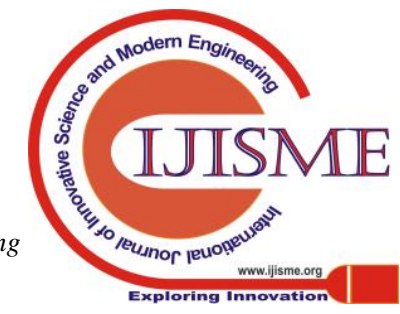




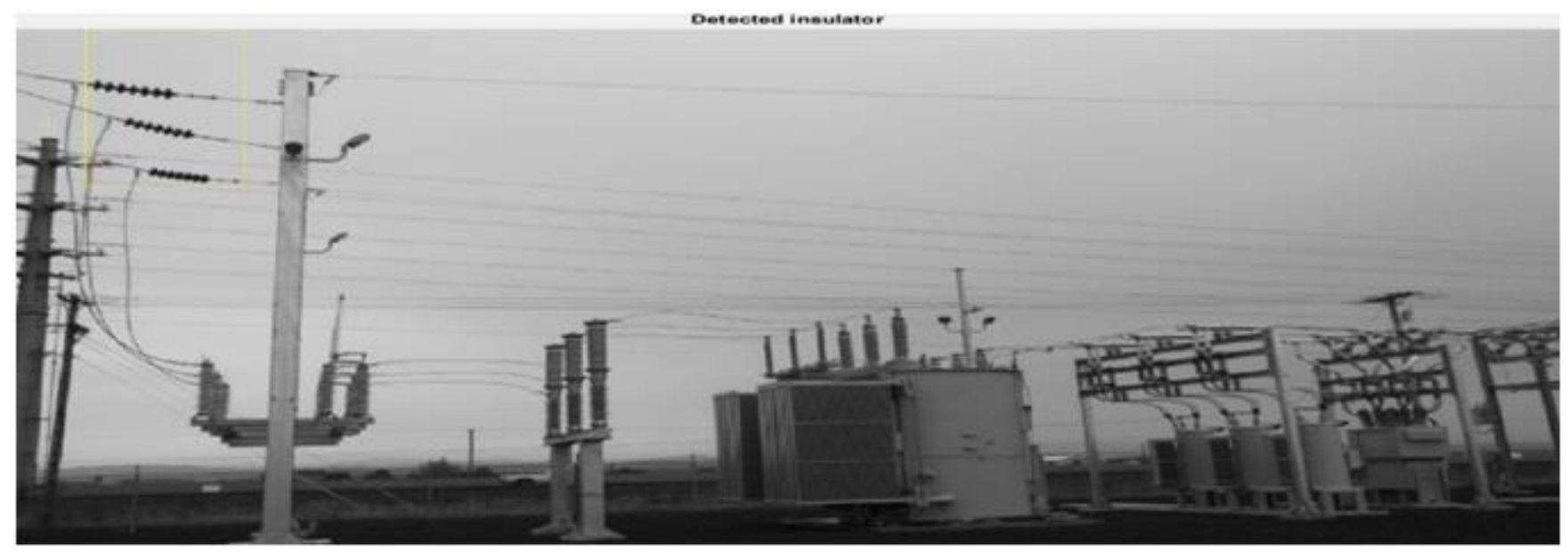

Fig. 13 Location of insulator in a cluttered scene

Fig.12 show the matched point including inliers only after the feature detection. The bounding of the object is done after this process in order to show the particualr image by bounding edges. Here rectangle polygon is used to show the object in the cluttered scene or substation image. The corner points are tranfered to the substation image, which will show the insulator object surrounded with the rectangle polygonFig.13 show the located object that is insulator in a cluttered scene or substation image on comparison with the reference image of insulator.

\section{CONCLUSION}

In this paper, we propose a Bag of Visual Word algorithm based image classification and Point Feature Matching algorithm for object detection towards insulator image datasets and substation image for insulator detection. The key techniques of our method include formation of visual vocabulary from traning images and use the extracted information by SURF features to classify new unlabeled images. This approach gives very good results with $90 \%$ of accuracy for large number of image datasets also. The point feature matching algorithm is based on SURF feature is used, which locate a object in a cluttered scene show better results than old feature descriptors based algorithm. The test results affirm its viability.

\section{REFERENCES}

1. Olivier Lezoray,Christophe Charrier, Hubert Cardot, and SebastienLef'evre, "Machine Learning in Image Processing," EURASIP Journal on Advances in Signal Processing, p. 2, 2008.

2. Haiyan Wang and Fanwei Meng, "Research on power equipment recognition method based on image processing," EURASIP Journal on Image and Video Processing, pp. 1-11, 2019 April.

3. Yongjie Zhai, Rui Chen, Qiang Yang, Xiaoxia Li,Zhenbing Zhao, "Insulator Fault Detection Based on Spatial Morphological Features of Aerial Images"," IEEE Access, vol. 6, pp. 35316-35326, 2018.

4. S. Lazebnik,C.Schmid, J. Ponce "Beyond bags of features: Spatial pyramid matching for recognizing natural scene categories," IEEE Computer Society Conf. on Computer Vision and Pattern Recognition, vol. 2, pp. 2169-2178, 2006.

5. J.Sivic, A. Zisserman, "Video Google: A Text Retrieval Approach to Object Matching in Videos," Proceedings of the Ninth IEEE International Conference on Computer Vision (ICCV), vol. 2, 2003.

6. $\quad$ K. M, C. Schmid, "A Performance Evaluation of Local Descriptors," IEEE TRANSACTIONS ON PATTERN ANALYSIS AND MACHINE INTELLIGENCE, vol. 27, no. 10, pp. 1615-1630, october 2005.

7. Y.-G. J. and C.-W. N, Wanlei Zhao, "Keyframe Retrieval by Keypoints: Can Point-to-Point Matching Help?," In Proc. of 5th Int'l Conference on Image and Video (CIVR), vol. 2006, pp. 72-81, 2006.

8. P. P. Li Fei-Fei, "A Bayesian Hierarchical Model for Learning
Natural Scene Categories," In Proc. of the 2005 IEEE Computer Society Conf. on Computer Vision and Pattern Recognition, pp. 524531, 2005.

9. R. A. Sameer, Abdul Amir Abdullah Karim, "Image Classification Using Bag of Visual Words (BoVW)," Al-Nahrain Journal of Science , vol. 21, no. 4, pp. 76-82, December 2018.

10. Y.G. Jiang, A. G. Hauptmann, C.W. Ngo, Jun Yang, "Evaluating Bag-of-Visual-Words Representations in Scene Classification," Proceedings of the International Workshop on Workshop on Multimedia Information Retrieval - MIR '07, pp. 197-206, 2007 September.

11. Dr. Y. J. V. Raj, D. Latha, "A Review on Different Categories of CBIR Methods," International Journal of Scientific Research in Computer Science, Engineering and Information Technology, vol. 3, no. 1, pp. 1093-1100, January-Feburary-2018

12. Mrs. J. D. Dhande, Miss Kirti Bhure, "Object Detection using SURF features," International Research Journal of Engineering and Technology (IRJET), vol. 04, no. 07, pp. 1524-1526, july 2017.

13. Z. Daixian, "'SIFT algorithm analysis and optimization,"," International Conference on Image Analysis and Signal Processing, pp. 415-419, 2010

14. Q. W. a. Y. C. Zetao Jiang, "A Fast Method for Feature Matching Based on SURF," International Conference on Intelligent Science and Intelligent Data Engineering, vol. 7202, pp. 374-381, 2012.

15. R. I. M. F. B. Faishal Badsha, "Object Detection by Point Feature Matching using Matlab," AVIP ADVANCES IN IMAGE AND VEDIO PROCESSING, vol. 6, no. 6, pp. 22-29, December 2018.

16. N. H. D. a. N. D. Georganas, "'Real-Time Hand Gesture Detection and Recognition Using Bag-of-Features and Support Vector Machine Techniques,"," IEEE Transactions on Instrumentation and Measurement, vol. 60, no. 11, pp. 3592-3607, Nov. 2011.

17. N. Pise. M. Phatak. Jasmine Irani, "Clustering Techniques and the Similarity Measures used in Clustering: A Survey," International Journal of Computer Applications, vol. 134, no. 7, pp. (0975-8887), january 2016.

18. A. Ess, T. Tuytelaars, L. V. Gool,. Herbert Bay, "Speeded-Up Robust Features (SURF)," Computer Vision and Image Understanding, ELSEVIER, no. 110, p. 346-359, december 2008.

19. T. LINDEBERG, "Feature Detection with Automatic Scale Selection," International Journal of Computer Vision, vol. 30, no. 2, pp. 76-116, july30, 1998.

20. D. G. Lowe, "Distinctive Image Features from Scale-Invariant Keypoints," International Journal of Computer Vision, January 5, 2004.

21. O. Cham, M. Urban, T. Pajdla, J. Matas, "Robust Wide Baseline Stereo from Maximally Stable Extremal Regions," Image and Vision Computing, 2004

22. C. S. KRYSTIAN MIKOLAJCZYK, "Scale \& Affine Invariant Interest Point Detectors," International Journal of Computer Vision (IJCV), vol. 60, no. 1, pp. 63-86, 2004.

23. D. G. Lowe, "Object Recognition from Local Scale-Invariant Features," in The International Conference on Computer Vision, corfu, September, 1999.

Published By:

Blue Eyes Intelligence Engineering

\& Sciences Publication 
24. S. R. Panchal, S. K. Shah, P M Panchal, "A Comparison of SIFT and SURF," International Journal of Innovative Research in Computer and Communication Engineering , vol. 1, no. 2, pp. 323-327, April 2013.

25. Sudipta M, P. K. Biswas, A. K. Dhara, M. K. Madaiah, N. Khandelwal, Chanukya Krishna Chamaa, "Automated Lung Field Segmentation in CT images using Mean Shift Clustering and Geometrical Features," in SPIE Medical Imaging: Computer-Aided Diagnosis, 2013.

26. Dr M. R.Daliri, A. Nodehi, A. Qorbani, A.Ahmadi, "Objects Recognition Using the Histogram Based on Descriptors of SIFT and SURF," Journal of Basic and Applied Scientific Research, 2012.

\section{AUTHORS PROFILE}

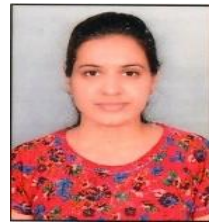

Ayushi Jadia, Research Scholar in Power system and Control, Electrical Engineering Department SGSITS, 23 Park Road Indore (M.P) India. She received her Bachelor Degree in Electrical Engineering from Vindhya Institute of Technology and Science, Satna (M.P) in 2018. Her research interests include Machine learning, Image Processing, Insulators recognition, Classification and Detection of electrical equipment.

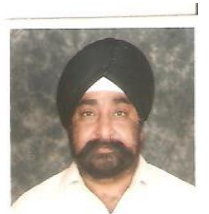

Prof. M.P. S Chawla, Professor-Incharge (Head), Library, Associate Professor in Electrical

Engg.SGSITS Indore (MP) India. Immediate Past Chairman, 2017-2018, IEEE MP Sub-Section. He received Gold Medals in BE (Electrical) and $\mathrm{ME}$ (Power Electronics) degrees SGSITS, Indore, India, from Departments of Electrical Engineering in 1988 and 1992, respectively. He is Appointed as "Associated EDITOR in Chief Chair", on 14 $4^{\text {th }}$ DEC2018, in Blue Eyes Intelligence Engineering and Sciences Publication, India.His special research interests are in Power Electronics, Devices, Intelligent Instrumentation, Biomedical Engineering, Signal Processing, Advanced Instrumentation Systems, Soft Computing, Higher Order Statistical Techniques and Control Systems 